\title{
Morbilidad Materna Extrema (MME): descripción de una serie de 23 casos y análisis de las "tres demoras"
}

\section{Extreme maternal morbidity (EMM): description of a 23-case series and Analysis of the "three delays"}

\author{
Julio César Quintero ${ }^{1}$, Sandra Olaya ${ }^{2}$, Aleida Peña², Yeiny Gil², Yolanda Palencia² \\ 1 MD., M.Sc. Profesor Especialización en Gerencia y Auditoría de la Calidad en Salud, Universi- \\ dad Jorge Tadeo Lozano. Neiva, Colombia. \\ 2 Especialistas en Gerencia y Auditoría de la Calidad en Salud, Convenio UTADEO - USCO. Nei- \\ va, Colombia. \\ Presentado en el Congreso Internacional de Investigación en Salud “Equidad y Acceso al Cuidado \\ Integral”. Correspondencia: Julio César Quintero Vieda, Correo electrónico: julioc.quinterov@uta- \\ deo.edu.co
}

\section{Resumen}

La Morbilidad Materna Extrema (MME) es un problema de Salud Pública que ha llamado la atención de las instituciones, agencias y gobiernos en todo el mundo por su alta frecuencia e impacto. A pesar de los esfuerzos, no se han logrado alcanzar las metas planteadas.

Objetivo: Caracterizar las demoras o retrasos en la atención en salud de pacientes con MME como factores determinantes de la presencia de estos eventos.

Métodos: Estudio descriptivo de serie de casos, en gestantes con MME durante el mes de Marzo de 2013 en un Hospital Universitario.

Resultados: En el periodo descrito se presentaron 23 casos de MME, el 65.2\% eran menores de 25 años. La mayoría residían en Cabecera Municipal (65.2\%), tenían una unión estable (65.22\%) y contaban con afiliación al régimen de salud (86.96\%). Se encontró una razón de 1.1 en cuanto a la gestación deseada versus no deseada, el $75 \%$ de las pacientes que no deseaban su embarazo, no utilizaban ningún método de anticoncepción. Ninguna de las pacientes asistió a consulta preconcepcional, la mitad tuvieron adherencia al programa de control prenatal y tan solo el $65.2 \%$ tuvieron una captación temprana.

Se identificaron principalmente demoras tipo uno (69.6\%) y tres (65.2\%). En ocho de los 23 casos (34.78\%) se presentó algún tipo de evento adverso; de estos, el $50 \%$ (cuatro casos) se clasificaron entre leve y moderado y el otro $50 \%$ como severos. 
Palabras clave: Complicaciones del Embarazo, Servicios de Salud Materna y Mortalidad Materna. (Fuente: $\mathrm{DeCS})$

\begin{abstract}
Extreme maternal morbidity is a public health problem that has been the focus of institutions, agencies and governments all over the world due to its high frequency and impact. Despite efforts made, established goals have not been reached.
\end{abstract}

Objective: to characterize delays in health care in patients with EMM as determining factors in the occurrence of these events.

Methods: a descriptive case series study with pregnant women suffering from EMM during March, 2013 at a University Hospital.

Results: there were 23 cases of women suffering from MME, $65.2 \%$ under 25 years of age. Most of them lived in urban areas (65.2\%), had a stable relationship (65.22\%) and had social security (86.96\%). A 1.1 ratio was found regarding wanted gestation against unwanted, $75 \%$ of patients who did not want their pregnancy, did not use any contraceptive method. None of the patients attended preconceptional medical consultation, half of them had adherence to prenatal program and just $65.2 \%$ had early uptake.

It was mainly identified type-one delays (69.6\%) and type three (65.2\%). In 8 (34.78\%) out of 23 cases there was some kind of adverse event; from these, $50 \%$ (four cases) were classified as mild and moderate and the remaining $50 \%$ was severe.

Keywords: pregnancy complications, maternal health care services and maternal mortality.

\section{Introducción}

Según estimaciones de la Organización Mundial de la Salud (OMS), cada año 210 millones de mujeres en todo el mundo quedan embarazadas, de las cuales unos ocho millones padecen complicaciones potencialmente mortales relacionadas con el embarazo ${ }^{(1)}$. La MME definida como "la complicación grave que ocurre durante el embarazo, parto y puerperio, que pone en riesgo la vida de la mujer o requiere de una atención inmediata con el fin de evitar la muerte(2)", integrada al estudio y análisis de la mortalidad materna, incrementa la oportunidad de identificar los factores que contribuyen a este proceso y permite tomar correctivos de una manera más oportuna ${ }^{(3-7)}$. La utilidad de este sinergismo radica en: a) los casos de MME son más frecuentes que los casos de muerte $\left.{ }^{(3-7)}, b\right)$ las lecciones aprendidas en el tratamiento de los casos que sobrevivieron se pueden usar para evitar nuevos casos de muerte materna, c) el análisis de los casos de MME son menos amenazantes para los proveedores de salud, d) existe la posibilidad de contar con la fuente primaria de información "la gestante sobreviviente" y e) su estudio se puede utilizar como una medida de evaluación de la calidad de los programas de control prenatal o para estudio de $\operatorname{casos}^{(8-10)}$.

La MME comprende un conjunto de complicaciones severas, prevenibles en su mayoría (11), su prevención depende principalmente del tipo y aplicación de la tecnología disponible, la calidad de la atención y el patrón de uso de los servicios ${ }^{(11,12)}$. De acuerdo con revisiones sistemáticas realizadas, se estima que la prevalencia mundial de complicaciones que amenazan potencialmente la vida de la mujer gestante, varía entre 0.3 y 101.7 casos por cada 1000 nacidos vivos, con una media de 8.2 casos por 1000 nacidos vivos ${ }^{(3,6)}$.

Los factores determinantes para la presencia de un caso de MME son básicamente tres: las condiciones de riesgo intrínsecas (factores endógenos de la madre y producto de la gestación) tales como multiparidad, edades extremas, adolescencia, 
entre otros ${ }^{(12,13)}$; el grado de vulnerabilidad (carencia o limitación de una condición protectora) tales como aspectos socioeconómicos, culturales, educativos, entre otros; y los factores sanitarios referido a debilidades en la organización de los servicios de salud que determinan limitaciones en el acceso, la disponibilidad, la oportunidad y calidad de la atención ${ }^{(12,14)}$.

El análisis de los resultados de diversas investigaciones realizadas $^{(2,7-11,15,16)}$, corrobora lo señalado por la OMS, respecto a que estas muertes son el resultado de un conjunto de factores que al entrelazarse forman una cadena fatal de "retrasos o demoras" que impiden prevenir riesgos durante los embarazos y que las mujeres accedan a servicios de salud de calidad. Según Maine, para reducir sustancialmente la MME y las muertes maternas en el futuro cercano, se debe analizar detalladamente la "cadena causal" de cada caso ${ }^{(15)}$. "Primero, la mujer debe estar embarazada, segundo, tiene que desarrollar un problema médico y tercero, para que la mujer muera, la complicación debe ser tratada inadecuadamente o no tratable" (15). Por consiguiente, para poder reducir la MME y por ende la mortalidad materna, cualquier intervención propuesta debe finalmente: reducir las posibilidades de embarazarse, reducir las posibilidades de que la mujer embarazada experimente graves complicaciones durante el embarazo o parto y/o reducir las posibilidades de muerte entre mujeres que experimenten complicaciones ${ }^{(15)}$.

De acuerdo con todo lo anterior, el presente trabajo pretende caracterizar las demoras o retrasos en la atención en salud de pacientes con MME como factores determinantes de la presencia de estos eventos en el departamento del Huila.

\section{Materiales y métodos}

Estudio descriptivo de serie de casos. La población objetivo fueron la totalidad de las gestantes o puérperas que ingresaron al Hospital de alta complejidad y de referencia del departamento del Huila, en el periodo comprendido entre el
01 y 31 de marzo del 2013, con una muestra formada por todas las pacientes que cumplieron con la definición de caso y los criterios de inclusión propuestos por la Federación Latinoamericana de Sociedades de Obstetricia y Ginecología (FLASOG), incluidos en el protocolo de vigilancia en salud pública para la MME del Instituto Nacional de Salud (INS) ${ }^{(8)}$.

La identificación de los casos se realizó por búsqueda activa mediante el seguimiento diario de las pacientes de nuestra población objetivo, a cada caso seleccionado se le realizó monitorización de las actividades efectuadas durante el tratamiento de su(s) complicación(es), al momento de su egreso se aplicó el instrumento "entrevista a la gestante sobreviviente" del INS ${ }^{(16)}$ y se reconstruyó cronológicamente todo el proceso de atención en salud, desde el momento en que se entera de su embarazo hasta la fecha de su egreso. A partir de esta información, se unificó la historia clínica de cada paciente, mediante requerimientos a las Instituciones que tuvieron contacto con ellas y se llevó a cabo una auditoría detallada de todos los registros clínicos existentes por cada paciente.

Cada caso fue analizado por los autores de acuerdo al protocolo de vigilancia y control de la MME del Instituto Nacional de Salud (INS) (16). Si bien hay numerosos factores que contribuyen a la mortalidad materna y por ende a la MME, nuestra investigación se centró en aquellos descritos por Maine et al. en $1997^{(15)}$. Se examinan, entonces, aquellos factores que: primero, retrasan la decisión de buscar ayuda; segundo, producen retraso en llegar a la instalación de tratamiento y; tercero, demoran la prestación de una atención adecuada. Estos retrasos fueron agrupados en tres etapas dentro del proceso de atención.

Demora en decidir buscar atención (Tipo uno): depende de las capacidades y oportunidades de la mujer y su entorno para reconocer una complicación que amenaza la vida, así como de la información de la que dispone acerca de a dónde puede acudir. 
Demora en identificar y acceder a un servicio de salud (Tipo dos): depende de la distancia al servicio de salud, la disponibilidad y eficiencia del transporte y el costo de los traslados.

Demora en obtener el tratamiento adecuado y oportuno (Tipo tres): depende de la disponibilidad de personal capacitado en la institución, la disponibilidad de medicamentos e insumos (antibióticos, sulfato de magnesio, sangre segura) y de la infraestructura (quirófanos) del servicio de salud $^{(15)}$.

Al realizar este estudio se pensó en caracterizar casos de MME desde un enfoque basado en procesos más que de análisis clínico.

Como variables de estudio se seleccionaron el perfil sociocultural de cada paciente (edad, área de procedencia, estado civil, régimen de afiliación en salud, nivel educativo y ocupación), su nivel de autodeterminación y planificación familiar (gestación deseada y método de planificación familiar utilizado), los factores de riesgos reproductivos preconcepcionales identificados y su concomitancia, las características de los puntos críticos más importantes del programa de control prenatal tomando como criterio de auditoría la guía de control prenatal y factores de riesgo de la Secretaría Distrital de Bogotá $^{(13)}$ y la norma técnica para la detección temprana de las alteraciones del embarazo de la resolución 412 de 2000 ${ }^{(17)}$ (asistencia a consulta preconcepcional, captación temprana antes de la semana 14, identificación oportuna de factores de riesgo y pertinencia en las medidas tomadas frente a estos y por parte del paciente adherencia a todas las actividades del programa e identificación oportuna de sus signos de alarma); las características de la atención y tratamiento de la MME tomando como punto de referencia las características del sistema obligatorio de garantía de la calidad en salud de nuestro país (accesibilidad, oportunidad, continuidad, pertinencia y seguridad $)^{(18)}$ y la guía de práctica clínica para la prevención, detección temprana y tratamiento de las compli- caciones del embarazo, parto o puerperio para profesionales de la salud del Ministerio de Salud de Colombia ${ }^{(19)}$ (oportunidad y pertinencia en las medidas tomadas por parte del equipo de atención frente a los signos de alarma identificados, continuidad en el proceso de referencia interinstitucional tanto hospitalario como ambulatorio, diagnóstico definitivo oportuno, pertinencia en el tratamiento instaurado medida como el grado de adherencia a la guía de referencia, continuidad en el proceso de atención y presencia de eventos adversos prevenibles a partir de las demoras o retrasos identificados tomando como referencia los lineamientos para la implementación de la política de seguridad del paciente del ministerio de salud de Colombia) ${ }^{(20)}$; y por último, haciendo un análisis general de los datos obtenidos se determinaron las demoras presentadas durante todo el proceso de atención.

Para la recolección y procesamiento de la información se diseñó una hoja Excel y un instructivo para su diligenciamiento, los datos fueron almacenados y analizados en una base de datos en Statistical Package for the Social Sciences (SPSS) for Windows, versión 18-0. La metodología de análisis fue cuantitativa (distribuciones de frecuencia, proporciones y modelo de las tres demoras).

En todos los casos se mantuvo la confidencialidad de la información recolectada. Para la presentación de los resultados se utilizaron tablas de distribución de frecuencia, este estudio contó con la revisión y aprobación del comité de ética en investigación del Hospital y se catalogó como Investigación sin riesgo.

\section{Resultados}

\section{Perfil sociodemográfico}

Durante el periodo de estudio se encontraron 23 casos de MME de un total de 540 pacientes ingresadas por causas obstétricas, en ese mismo 
mes se registraron 538 partos, 536 nacidos vivos y 3 muertes maternas.

Los pacientes con MME se caracterizaron desde el punto de vista socio-demográfico por tener una edad promedio de 24,6 años con desviación estándar de 6,53 y moda de 23 años, con edad mínima de 16 y una máxima de 36 años y el $65.2 \%$ de ellas eran menores de 25 años (Tabla 1).

\section{Autodeterminación y planificación familiar (PNF)}

Se encontró una razón de 1.1 en cuanto a gestación deseada vs no deseada, lo que significa que solo 11 (47.8\%) de las 23 pacientes deseaban su gestación, de éstas solo tres planificaban, dos con anticonceptivos inyectables y una con DIU, las restantes 9 pacientes manifestaron no utilizar algún método de anticoncepción.

\section{Riesgos reproductivos preconcepcionales}

Los factores de riesgo reproductivos preconcepcionales (RRPC) encontrados con mayor frecuencia en la muestra fueron las dificultades para el acceso a los servicios de salud $(45,4 \%)$, bajo nivel de escolaridad $(40,9 \%)$, antecedente de cirugía uterina $(22,7 \%)$ y madre soltera (22,7\%); al revisar el número de RRPC identificados a cada paciente encontramos que solo dos pacientes $(8.7 \%)$ no presentaban ningún factor de riesgo, dos más se les identificaron más de tres factores de riesgo concomitantes y al resto $(82.6 \%)$ se les identificaron uno, dos o tres factores de riesgo al inicio del control prenatal.

\section{Características del control prenatal (CPN)}

Se encontró como hallazgos positivos la identificación oportuna de factores de riesgo y pertinencia en las medidas tomadas frente a estos, en

Tabla 1. Distribución por área de procedencia, estado civil, régimen de afiliación en salud, nivel educativo y ocupación de las maternas con MME

\begin{tabular}{lccc}
\hline \multicolumn{1}{c}{ Variable } & Escala & $\mathbf{N}$ & $\mathbf{P ~ ( \% )}$ \\
\hline \multirow{3}{*}{ Área de procedencia } & Cabecera municipal & 15 & 65.2 \\
& Centro poblado & 2 & 8.7 \\
& Rural disperso & 6 & 26.1 \\
\hline \multirow{3}{*}{ Estado civil } & Soltera & 3 & 13 \\
& Casada & 5 & 21,7 \\
& Unión estable & 15 & 65,2 \\
\hline \multirow{2}{*}{ Régimen de afiliación } & Contributivo & 6 & 26,1 \\
& Subsidiado & 14 & 60,9 \\
& Especial & 1 & 4,3 \\
& Vinculado & 1 & 4,3 \\
& No afiliado & 1 & 4,3 \\
\hline \multirow{2}{*}{ Nivel educativo } & Analfabeta & 1 & 4,3 \\
& Primaria & 8 & 34,8 \\
& Secundaria & 13 & 56,5 \\
Ocupación & Universitaria & 1 & 4,3 \\
\hline
\end{tabular}


el $82.6 \%$ y $100 \%$ de los casos respectivamente. Preocupa que ninguna de las gestantes contó con consulta preconcepcional, solamente la mitad tuvieron adherencia al programa de CPN, tan solo el $65.2 \%$ tuvieron una captación temprana al mismo y el 69.6\% una identificación oportuna por parte de la paciente de sus signos de alarma.

\section{Características de la atención en salud de las maternas con MME}

Posterior a la respectiva solicitud de atención por parte de la paciente, se evaluaron las características de esa primera atención encontrando oportunidad y pertinencia en las medidas tomadas por parte del equipo de atención frente a los signos de alarma identificados, en el 68,2\% y $72,7 \%$ de los casos respectivamente. Solamente dos gestantes $(8.7 \%)$ presentaron demoras en el proceso de referencia interinstitucional.

Luego de la atención inicial, se evaluó el proceso de investigación diagnóstica realizado y el tratamiento definitivo instaurado, identificando que el $56.5 \%$ de los casos tuvieron diagnóstico definitivo oportuno y el $60.9 \%$ pertinencia en el tratamiento; respecto a la continuidad de la atención brindada el $39.1 \%$ de la pacientes presentaron problemas administrativos que retrasaron su atención, por lo demás en el 91.3\% no se presentaron inconvenientes en cuanto a insumos o medicamentos, personal asistencial suficiente y disponibilidad de camas en unidad de cuidados intensivos, tanto neonatal como obstétricas.

\section{Análisis de las "tres demoras"}

En las pacientes con MME se identificaron demoras tipo uno y tres con un $69.6 \%$ y $65.2 \%$ respectivamente, el $47.8 \%$ presentaron demora tipo dos; solo una paciente no presentó algún tipo de demora (4.3\%), a la gran mayoría de las pacientes se les identificaron dos tipos diferentes de demoras $(60.9 \%)$ y al resto se le identificaron las tres demoras durante todo su proceso de atención o un solo tipo de demora con un porcentaje de $13.0 \%$ y $21.7 \%$ respectivamente.

\section{Eventos adversos identificados durante el pro- ceso de atención de las maternas con MME}

En el $34.78 \%$ de los 23 casos se presentó algún tipo de evento adverso durante cualquiera de las etapas de la atención de estas pacientes; de estos, el 50\% (cuatro casos) se clasificaron entre leve y moderado y el otro 50\% como severo.

\section{Discusión}

El periodo de estudio arrojó un total de 23 casos de MME que ingresaron al Hospital en el periodo de estudio, resultado congruente con el histórico institucional y las características de la Institución.

Según la Encuesta Nacional de Demografía y Salud (ENDS) del $2010^{(21)}$, en Colombia el conocimiento de los métodos de PNF es universal, el $81,6 \%$ de las mujeres entre 15 y 49 años sexualmente activas no unidas hacen uso de métodos anticonceptivos y su uso entre mujeres en unión, en el área urbana es de 79\% y en el área rural de $79,2 \%{ }^{(21,22)}$; al confrontar nuestros resultados, en donde se encontró que cerca de la mitad de las pacientes estudiadas no deseaban su embarazo, podemos inferir que existen bajos niveles de impacto de los programas de promoción de la autodeterminación sexual y PNF a nivel regional que amerita una investigación más profunda en esta materia, en especial en pacientes con antecedentes obstétricos adversos o factores de riesgo declarados, las cuales no han sido adecuadamente priorizadas para este tipo de actividades.

Respecto a las características de los programas de control prenatal, en primer lugar, se estableció que la captación temprana (antes de la semana 14) es deficiente lo que dificulta la intervención oportuna de los riesgos preconcepcionales y deja de manifiesto un claro problema de demanda inducida en este tipo de programas, au- 
nado a la baja demanda natural e inasistencia al CPN en el primer trimestre asociada a factores propios de la paciente ${ }^{(23)}$; en segundo lugar, se observó baja adherencia por parte de las pacientes a las actividades del programa, solo el $65,2 \%$ de las pacientes que tuvieron un embarazo mayor de 37 semanas de gestación tuvieron más de 4 controles prenatales, el 21,7\% menos de 4 y el 8,7\% no tuvieron controles; al comparar estos datos con las cifras oficiales del observatorio de MME de la Secretaría de Salud Departamental del Huila en el 2011, en las que el 74\% de las pacientes con MME tuvieron más de 4 controles prenatales, podemos observar que no existen diferencias significativas.

La identificación oportuna de signos de alarma, muestra buena gestión respecto a las actividades de educación en salud de la madre y su familia durante los diferentes contactos que las pacientes tienen con los servicios de salud.

Luego de identificados los signos de alarma, el acceso a las diferentes etapas del proceso de atención es oportuno y continuo en la gran mayoría de los casos, a pesar de lo anterior, los resultados dejan abierta una brecha de desempeño que sugiere la existencia de fallas en los procesos integrales de atención, que deben ser estudiados con mayor profundidad para lograr su mitigación y en un futuro no muy lejano su eliminación.

Respecto al estudio de la pertinencia y la seguridad clínica, tanto la oportunidad en la ejecución del proceso de investigación diagnóstica, como la adherencia a las guías de referencia, mostraron niveles altos de cumplimiento, pero llama la atención que el 43,5\% de los casos hayan tenido algún tipo de demora en los procesos de identificación de complicaciones presentadas durante el proceso de atención, tales como retrasos en la identificación de hemorragias postparto o postcesarea, infecciones asociadas al cuidado de la salud, entre otras; sumado a lo anterior, también se evidenció inoportunidad en la realización de ayudas diagnósticas y problemas administrativos que retrasan el proceso de atención, hechos que obligatoriamente redundan en la oportunidad del diagnóstico de las complicaciones.

Respecto a los niveles de seguridad clínica, al comparar los datos obtenidos en el presente estudio con los resultados del estudio de Prevalencia de Efectos Adversos en Hospitales de Latinoamérica (IBEAS) ${ }^{(24)}$, en el cual la prevalencia de pacientes con al menos un EA fue de 10,5\% y la incidencia fue de 19,8\% en la población estudiada, y teniendo en cuenta que nuestro estudio no pretende estimar la incidencia o la prevalencia de estos eventos y que tomamos para el análisis pacientes con gran susceptibilidad a sufrir de estos eventos, se dejan abiertas muchas preguntas a resolver sobre la relación de causalidad existente entre los factores asociados a la génesis de los casos de MME y la presencia de eventos adversos prevenibles en nuestras Instituciones y viceversa.

Luego de un análisis general de los datos obtenidos se se logró identificar principalmente demoras tipo uno y tres; al comparar nuestros resultados con otras fuentes locales de esta información, encontramos que de acuerdo con el observatorio de MME del departamento del Huila las demoras tipo uno y dos son las más frecuentes, diferencia tal vez justificada por el sistema de vigilancia activa de los casos y el análisis paso a paso del proceso de atención realizado en este trabajo ${ }^{(25)}$.

Este estudio tiene algunas limitaciones relacionadas con su diseño metodológico (descriptivo, unicéntrico, entre otros), a pesar de la pequeña muestra obtenida, la profundidad en el estudio de cada caso desde un enfoque sistémico orienta a la identificación de los retrasos o demoras en la atención de este tipo pacientes y a la caracterización de un nuevo perfil y delimitación más integral de este tipo de eventos. 


\section{Bibliografía}

1. Organización Mundial de la Salud. Estrategia de salud reproductiva para acelerar el avance hacia la consecución de los objetivos y las metas internacionales de desarrollo. Ginebra, Suiza.2004.

2. Federación Latinoamericana de Sociedades de Obstetricia y Ginecología, Organización Mundial de la Salud, Organización Panamericana de la Salud, Centro Latinoamericano de Perinatología y Desarrollo Humano. Comité de Mortalidad Materna. Santa Cruz de la Sierra, Bolivia.2007.

3. SAY L, PATTINSON RC, GÜLMEZOGLU AM. WHO systematic review of maternal morbidity and mortality: the prevalence of severe acute maternal morbidity (near miss). Reproductive health. 2004;1(1):3.

4. CECATTI JG, SOUZA JP, PARPINELLI MA, DE SOUSA MH, AMARAL E. Research on Severe Maternal Morbidities and Near-Misses in Brazil: What We Have Learned. Reproductive Health Matters. 2007 11/01;15(30):125-33.

5. GALLEGO Vélez L, VÉLEZ Álvarez GA, AGUDELO Jaramillo B. Panorama de la Mortalidad Materna. Medellín, Colombia.2005. Available from: http://medicina.udea.edu.co/ Dependencias/Ginecologia/CLAP/pdf/libros/libro1/panoramadelamortalidadmaterna.pdf.

6. SOUZA JP, CECATTI JG, PARPINELLI MA, DE SOUSA MH, SERRUYA SJ. Revisão sistemática sobre morbidade materna near miss [Systematic review of near miss maternal morbidity]. Cad Saúde Pública= Rep Public Health. 2006;22(2):255-64.

7. GELLER SE, ROSENBERG D, COX SM, BROWN ML, SIMONSON L, DRISCOLL $\mathrm{CA}$, et al. The continuum of maternal morbidity and mortality: factors associated with severity. Am J Obstet Gynecol. 2004 Sep;191(3):939-44.

8. ORTIZ EI, QUINTERO CA, MEJÍA J, ROMERO E, OSPINO L. Vigilancia de la Morbilidad Materna Extrema. Bogotá, Colombia.2010.

9. REYES-ARMAS I, VILLAR A. Morbilidad materna extrema en el Hospital Nacional Docente Madre-Niño San Bartolomé, Lima, 20072009. Rev peru ginecol obstet. 2012;58(4):273-84.
10. HOLDEN DA, QUIN M, HOLDEN DP. Clinical risk management in obstetrics. Current Opinion in Obstetrics and Gynecology. 2004;16(2):137-42.

11. DANEL I, BERG C, JOHNSON CH, ATRASH H. Magnitude of Maternal Morbidity During Labor and Delivery United States, 1993-1997. Obstetrical \& Gynecological Survey. 2003;58(9):572-3.

12. World Health Organization. Beyond the Numbers: Reviewing maternal deaths and complications to make pregnancy safer. Genova, Suiza.2004.

13. Secretaría Distrital de Salud de Bogotá D.C., Asociación Bogotana de Obstetricia y Ginecología (ASBOG). Guía de control prenatal y factores de riesgo. Bogotá D.C., Colombia.2005.

14. CARRILLO UA. Mortalidad materna en Colombia: reflexiones en torno a la situación y la política pública durante la última década. Revista Ciencias de la Salud. 2007;5:72-85.

15. MAINE D, AKALIN MZ, WARD V, KAMARA A. Diseño y evaluación de programas para mortalidad materna. Nueva York, EUA.1997.

16. Instituto Nacional de Salud, Grupo de vigilancia y control de enfermedades no transmisibles. Protocolo de vigilancia y control de la morbilidad materna extrema. Bogotá, Colombia.2012.

17. Resolución número 412 de 2000 (febrero 25). Por la cual se establecen las actividades, procedimientos e intervenciones de demanda inducida y obligatorio cumplimiento y se adoptan las normas técnicas y guías de atencion para el desarrollo de las acciones de protección especifica y detección temprana y la atención de enfermedades de interés en salud pública., Diario Oficial, No 43.956, (31-03-2000).

18. Decreto 1011 de 2006 (abril 03). Por el cual se establece el Sistema Obligatorio de Garantía de Calidad de la Atención de Salud del Sistema General de Seguridad Social en Salud., Diario Oficial, No 46230, (03-04-2006).

19. Ministerio de Salud de Colombia. Guía de Práctica Clínica (GPC) para la prevención, detección temprana y tratamiento de las complicaciones del embarazo, parto o puerperio para profesionales de la salud. Bogotá, Colombia.2013. 
20. Ministerio de Salud de Colombia. Lineamientos para la implementación de la política de seguridad del paciente 2008.

21. Asociación Probienestar de la Familia Colombiana (Profamilia). Encuesta nacional en demografía y salud 2010. Bogotá, Colombia2010.

22. Ministerio de Salud y Protección Social. Análisis de Situación de Salud según Regiones. Bogotá, Colombia.2012.

23. CÓRDOBA R, ESCOBAR LP, GUZMÁN LL. Factores asociados a la inasistencia al primer trimetre del control prenatal en mujeres gestantes de la E.S.E. San Sebastián de la Plata (Huila). Revista Facultad de Salud - RFS Universidad Surcolombiana. 2012;4(1):39 - 49.

24. Ministerio de Sanidad y Política Social de España. Estudio IBEAS: Prevalencia de Efectos Adversos en Hospitales de Latinoamérica. Informe General. Madrid, España.2009.

25. Secretaría Departamental de Salud del Huila. Observatorio de Morbilidad Materna Extrema: Análisis descriptivo de 231 casos. Neiva, Colombia.2011. 